\title{
HuValue: A Toolkit to Facilitate Considering Various Human Values in a Design Process
}

\begin{abstract}
Human values play an integral role in any design that aims to improve the quality of human life. To support designers' addressing human values in their design, we created a design tool based on a comprehensive value framework. This tool helps to raise designers' awareness about human values and provide tangible materials to actively use selected values in a design process. An experimental study with design students showed that the project groups that had been supported with this tool addressed human values in their design concepts significantly stronger compared to the control groups. Results of the evaluation indicate that the tool is not only applicable in a design process but also effective at enriching design concepts with human values.
\end{abstract}

\section{Keywords}

Design Tool, Human Values, Value Framework, Design Process

\section{Introduction}

Nowadays, life without technology is hardly imaginable. Technology is interwoven with all aspects of life, and people perceive the world via artifacts [1, p. 235]. As artifacts are not self-formed phenomena, emphasizing on the role of these products in human's life brings the responsibility of designers in the light [1, p. 234]. Nevertheless, while design in its origin "aims at changing existing situations into preferred ones" [2, p. 111], there are very few agreements on what a preferred or better situation means and how it could be achieved. In this respect, human values can be introduced as a touchstone. In fact, values can be embedded in artifacts [3], and values expressed by the designer should be interpreted by the user [4]. We use these points to argue that considering human values behind every human action, goal and decision can provide a wide and sensitive view. This view has implications for design as the designer needs to understand what is preferable for the end-users and to make appropriate decisions [4]. Despite the significance of human values in everyday life and consequently, in design [5], they mostly remain implicit and unarticulated in design projects [6]. Only few design approaches concentrate on human values and aim to address them in their design: Value Sensitive Design (VSD) [7], Value-led Participatory Design (VPD) [8], and Value-Centred Design (VCD) [9]. Nonetheless, there is very little agreement between them to identify values. In this respect, the lack of an established and accepted fundamental grounding [10] and a comprehensive list of values [11] can be considered as a major unresolved issue. Accordingly, our research intended to support product designers considering human values consciously and explicitly in the design 
process. The core assumption in our study is that a wide view on human values and facilitating using this concept in design would help designers to take human values into account.

\section{Exploration of a Design Tool Based on a Value Framework}

Since the term 'value' is widely used for different purposes in various disciplines, and there is no comprehensive value framework for design, we considered the need for supporting product designers regarding human values: a general list of human values to cover diverse views as well as a wellclassified framework to summarize the list in a brief, understandable and applicable model and simplify thinking about and discussing them. A holistic view of the values of different aspects of human life [12] is important to improve the quality of humans' life, and this improvement is related to progress in all aspects of life [13]. Due to the natural distance between the

Fig. 1. Value wheel (Size: A2), with nine value clusters and fivepoint Likert-type scales. abstract level of human values and practical level of design, we considered the need for a tangible medium to bridge this gap and facilitate using a comprehensive value framework.

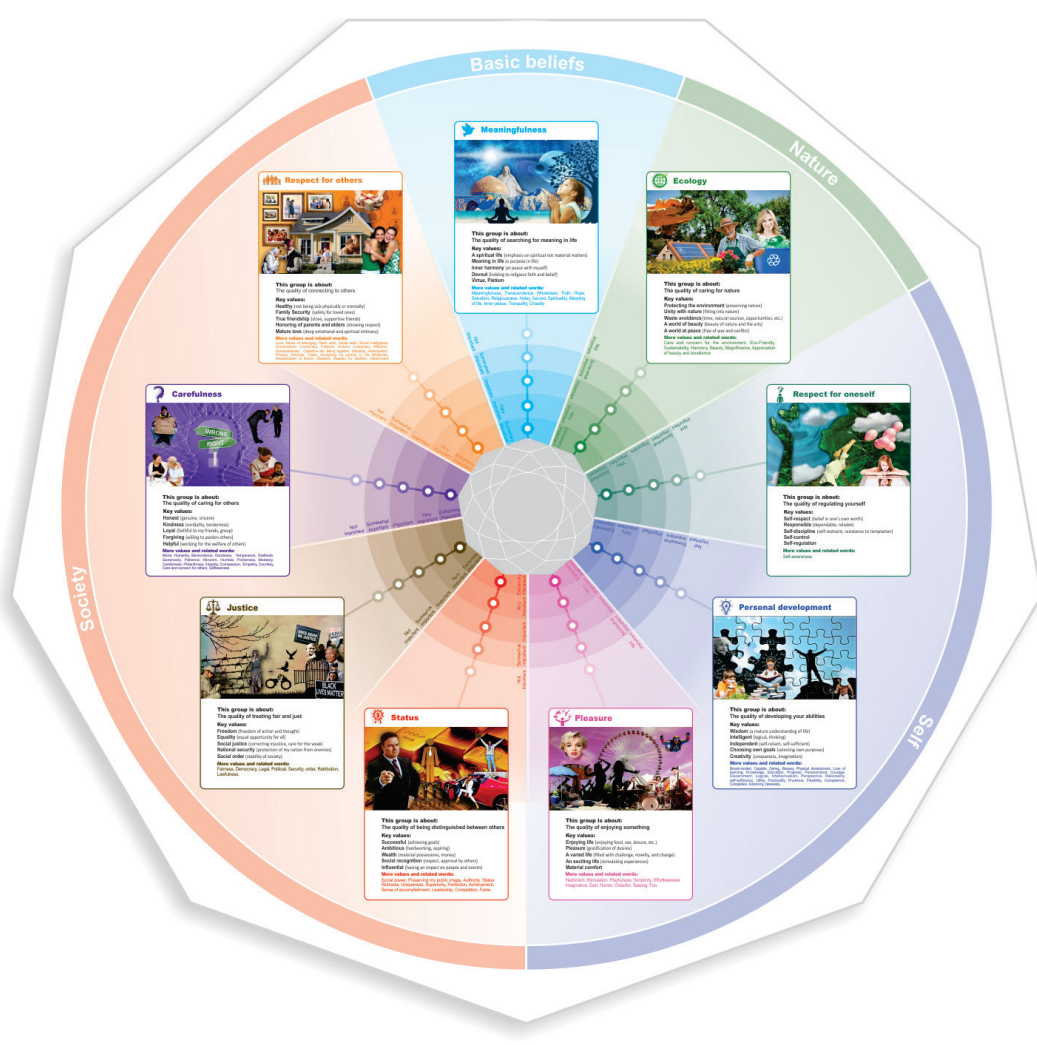

The HuValue tool is designed in the form of a cardbased design tool (Fig. 1 and Figure 2). The form of a card-based tool was selected for the design tool since cards are low-tech, tangible, and approachable design materials which are an effective medium to bridge the gap between theories and practice [14]. The HuValue tool contains a value wheel (Figure 1), 45 value words, and 207 picture cards (Figure 2). This tool is grounded on a comprehensive value framework for design [15]. This framework was created and developed via various theoretical, empirical and design-based approaches to compile, classify and structure the existing value lists, including Rokeach [16], Schwartz [17], Peterson and Seligman [18], and ten more value lists from the last century [15]. In the HuValue tool, the value framework is illustrated in a circular structure as the value wheel (Figure 1).

The Value wheel is a circle with nine value clusters, each with an icon, a label, a mood board, a descriptive sentence, five key values, and some relevant terms. This wheel provides an opportunity to express the importance of different human values, which can vary from person to person and situation to situation. The value clusters can be ranked in their order of importance via a 5-point Likert scale: 'Extremely important', 'Very important', 'Important', 'Somewhat important', and 'Not important'. The nine value clusters are 'Carefulness', 'Justice', 'Ecology', 'Respect for others', 'Meaningfulness', 'Status', 'Pleasure', 'Respect for oneself' and 'Personal development'. The outer circle indicates the relation of the value clusters to four general themes, including 'Basic believes', 'Nature', 'Self', and 'Society'.

The Value words are 45 two-sided cards $(5 \times 7 \mathrm{~cm})$ with a value word on the front and its relevant value cluster at the back [15]. These cards represent the key values mentioned in the value wheel to provide more flexibility for using and emphasizing individual values.

The Picture cards contain 207 cards $(7 \times 10 \mathrm{~cm})$ of three different types: 66 activities, 66 personae, and 75 products/services. Picture cards, as complementary to value wheel, are examples of activities, personas, and products/services that are presented in the format 

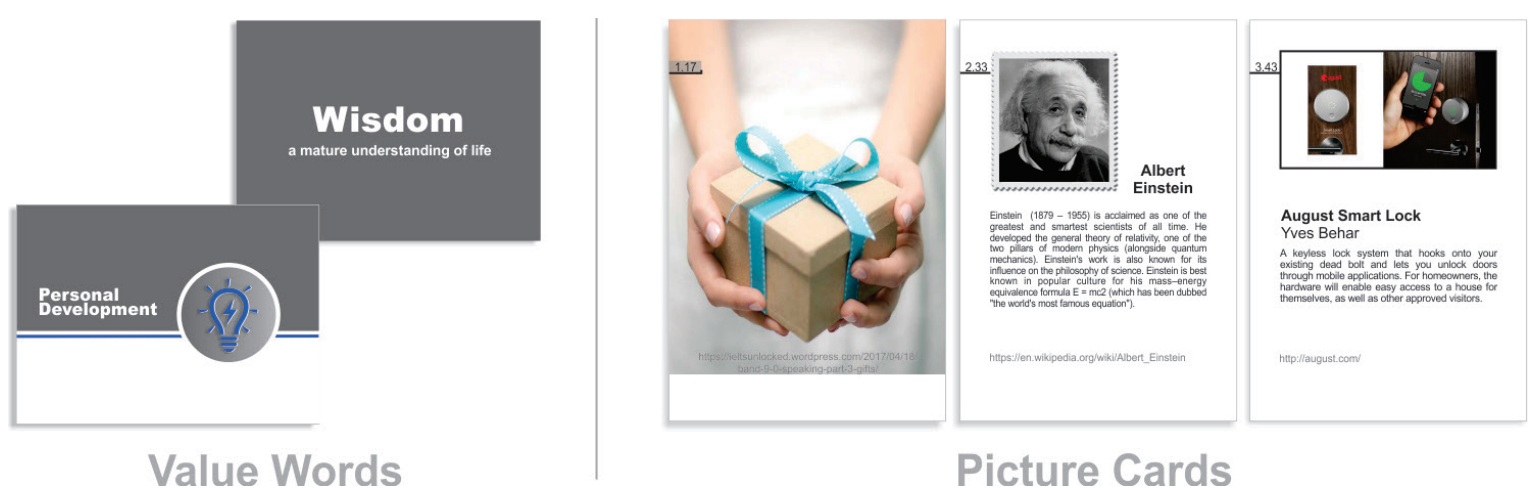

Fig. 2. Examples of value words and picture cards: (Left) two-sided cards with a value word on the front and its relevant value cluster at the back; (Right) three different types of picture cards including activity, persona, and product/service.

of cards. Despite the simplicity of their presentation, these cards' role is to link the abstract human values to everyday life. In fact, the cards are supposed to be applied for expressing human values in practice; the activity cards are some examples of the possible valuable behaviors, which can be used to express what does a specific value/group of values mean and how does it appear in a real life; The persona cards are the examples of iconic people, who can be representative of acting based on a specific value/group of values in life; and the products/services cards are some examples that can be used to try expressing how using a product/ service in daily life can straighten or weaken a value/ group of values.

Generally, the HuValue tool is a mean to facilitate thinking about and discussing human values. This tool supports designers with simple but familiar materials during their design process to analyze everything (object/subject/situation) from a wide value point of view. This approach, referred to as the HuValue perspective, enables the designers to be aware of and sensitive to human values and consider various aspects of their topic and different types of values.

Being aware of the diversity in design processes, the HuValue tool is intended to be used for several applications based on common design activities such as analyzing the situation, defining design goal, generating ideas, selecting a final idea, developing the concept, realizing and evaluating the final concept. In this respect, the tool can be applied for various purposes: the design challenge, the design goal, the context of use, and the user's needs, desires and requirements can be studied from a value-centered point of view for a better understanding of the design situation; human values can be used not only as a source of inspiration to diverge the ideas but also so that they cluster and converge; values can be seen as criteria for deciding on the final idea; the final concepts can be evaluated from a value perspective.

\section{Evaluation}

To investigate the value of the HuValue tool, a quasiexperimental study was realized to test the applicability and effectiveness of the tool in a design process [19]. A group of bachelor students ( $N=64$, out of 192 students) were randomly selected to take part in this study. After training them about the intended usage of the tool, the students were supposed to use the tool in their design projects. The data were collected via questionnaires and the students' final deliverables of their conceptual design. The outcomes showed that the project groups who were supported with this tool addressed significantly stronger human values in their design concepts compared to the control groups. Results of the evaluation indicated that the tool is not only applicable in a design process but is also effective at enriching design concepts with human values.

\section{Conclusion}

Considering the outcomes of our study, we can conclude that raising awareness about human values and facilitating using a value point of view seems to be 
helpful to emphasize human values in design. However, the current study is limited by time, and further investigations and developments should be realized. Next steps are to digitalize the tool and make it more simple and persuasive to use. We also recommend investigating how to apply the tool for changing behavior and how to use it for preserving and/or changing values.

\section{References}

1. Verbeek, P. P. (2005). What things do: Philosophical reflections on technology, agency, and design. (R. P. Crease, Trans.) Pennsylvania: Penn State Press.

2. Simon, H. A. (1996). The sciences of the artificial. Cambridge: MIT press.

3. van den Hoven, J., Vermaas, P. E., \& van de Poel, I. (Eds.). (2015). Handbook of ethics, values, and technological design. Dordrecht: Springer.

4. Le Dantec, C. A., \& Do, E. (2009). The mechanisms of value transfer in design meetings. Design Studies, 30(2), 119-137.

5. Kheirandish, S., \& Rauterberg, M. (2018). Human value based game design. In Proceedings of 2nd National and 1st International Digital Games Research Conference: Trends, Technologies, and Applications (DGRC) (pp. 6-16). Tehran, Iran: IEEE.

6. Steen, M., \& van de Poel, I. (2012). Making values explicit during the design process. IEEE Technology and Society Magazine, 31(4), 63-72.

7. Friedman, B., Hendry, D. G., \& Borning, A. (2017). A survey of Value Sensitive Design methods. Foundations and Trends $®$ in Human-Computer Interaction, 11(2), 63-125.

8. Iversen, O. S., Halskov, K., \& Leong, T. W. (2012). Value-led Participatory Design. CoDesign: International Journal of CoCreation in Design and the Arts, 8(2-3), 87-105.

9. Cockton, G. (2005). A development framework for valuecentred design. In Proceedings of CHI 2005 Extended Abstracts on Human factors in Computing Systems (pp. 1292-1295). Portland, Oregon, USA: ACM.

10. Boztepe, S. (2003). The notion of value and design. In $H$. Aoki (Ed.), In Proceedings of the Asian Design International Conference, 1 [CD]. Tsukuba, Japan.

11. Borning, A., \& Muller, M. (2012). Next Steps for Value Sensitive Design. In Proceedings of the SIGCHI Conference on Human Factors in Computing Systems 2012 (pp. 11251134). Austin, Texas: ACM.

12. Verkerk, M. J., Hoogland, J., van der Stoep, J., \& de Vri, M. J. (2016). Philosophy of Technology. An introduction for technology and business students. London: Routledge.

13. Saariluoma, P., Cañas, J., \& Leikas, J. (2016). Designing for Life. London: Palgrave Macmillan.

14. Mora, S., Asheim, J., Kjøllesdal, A., \& Divitini, M. (2016). Tiles Cards: a Card-based Design Game for Smart Objects Ecosystems. In Proceedings of the First International Workshop on Smart Ecosystems Creation by Visual Design, (p. 19-24). Bari, Italy: SERVE@AVI.

15. Kheirandish, S. (2018). HuValue - A tool to enrich design concepts with human values. (Doctoral dissertation). Eindhoven University of Technology, Eindhoven, The Netherlands.

16. Rokeach, M. (1973). The Nature of Human Values. New York: The Free Press.

17. Schwartz, S. (1992). Universals in the content and structure of values: Theoretical advances and empirical tests in 20 countries. Advances in experimental social psychology, 25 , 1-65.

18. Peterson, C., \& Seligman, M. (2004). Character strengths and virtues: A handbook and classification (Vol. 1). Oxford University Press.

19. Kheirandish, S., Funk, M., Wenswen, S., Verkerk, M., \& Rauterberg, M. (in press). HuValue: A tool to support design students in considering human values in their design. International Journal of Technology and Design Education. 